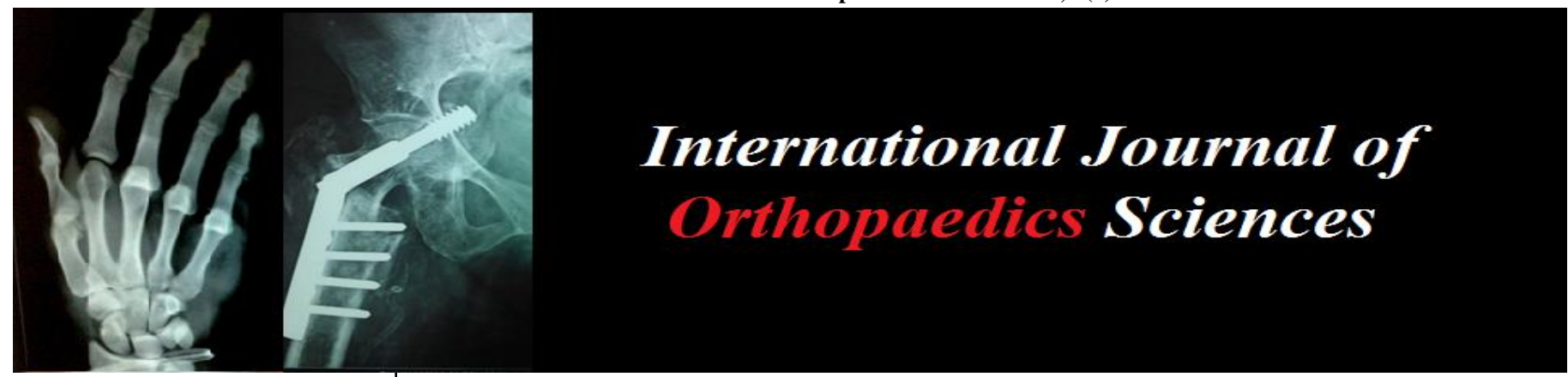

ISSN: $2395-1958$

IJOS 2018; 4(2): 555-557

(C) 2018 IJOS

www.orthopaper.com

Received: 21-02-2018

Accepted: 22-03-2018

Dr. Arshad AR

M.S Ortho Post Graduate, Department of Orthopaedics,

Sree Balaji Medical College and Hospital, BIHER, Works Road,

New Colony, Chromepet,

Chennai, Tamil Nadu, India

Dr Venkatachalam K

Professor, Department of

Orthopaedics, Sree Balaji

Medical College and Hospital,

BIHER, Works Road, New

Colony, Chromepet, Chennai,

Tamil Nadu, India
Correspondence

Dr Venkatachalam K

Professor, Department of

Orthopaedics, Sree Balaji

Medical College and Hospital,

BIHER, Works Road, New

Colony, Chromepet, Chennai,

Tamil Nadu, India

\section{Augmentative plate osteosynthesis with autologous bone grafting for aseptic non-union of femur with intramedullary interlocking nail in-situ: A prospective study}

\section{Dr. Arshad AR and Dr. Venkatachalam K}

DOI: https://doi.org/10.22271/ortho.2018.v4.i2i.82

Abstract

Intramedullary interlocking nailing has become the gold standard of treatment for femoral shaft fractures with union rate approaching $97 \%(1,2)$. In cases which have comminution at the fracture site however, interlocking nails alone fail to achieve union. This is specially true for fractures involving the metaphysis and metaphyseo-diaphyseal zone. The main reason for the resulting non-union is the failure of the interlocking nailing system not being able to provide adequate rotational stability of the fracture fragments. It is in these circumstances, that augmentative plate osteosynthesis along with autologous bone grafting gives sound union in 20 to 24 weeks. This prospective study was done on 24 patients who presented with aseptic non-union of the shaft of femur within 9 months of primary surgery, who had surgical augmentative plate osteosynthesis done along with autologous bone grafting and all went on to sound bony union, with an average union time of 22.7 weeks (range : 20 to 24 weeks).

Keywords: Aseptic non-union femur, interlocking nail, Augmentative plate osteosynthesis

\section{Introduction}

Intramedullary interlocking nailing is an accepted orthopaedic surgical procedure to treat femoral diaphyseal fractures. In most of the cases it results in sound union in about 5 to 6 months. In cases which have comminution at the fracture site, however interlocking nails alone fail to achieve union. This is especially true for the fractures involving the diaphyseometaphyseal or metaphyseal zone of the femur of the long bones. The main reason for the resulting non-union is the inability of the interlocking nailing system to provide for adequate rotational stability of the fracture fragments.

\section{Materials and method}

Our prospective study was conducted from JULY 2015 to JUNE 2017, involving 24 patients. We had recruited patients for 12 months (i.e from July 2015 to June 2016), so that there shall be a minimum 12 months period for follow up. All patients were initially treated with closed intramedullary interlocking nailing for the femoral shaft fractures. The mean interval between the primary interlocking nailing and the secondary augmentative plating with autologous bone grafting was 5 months (range: 4 months to 9 months).

\subsection{Inclusion criteria}

1. Both male and female patients in the age group 30 to 49 years of age were included in the study.

2. Only aseptic non-union presenting within 9 months of primary surgery were included in the study.

\subsection{Exclusion criteria}

1. Cases of nailing performed for pathological fractures and infected non-union were excluded from the study.

2. Smokers and patients addicted to tobacco related products were excluded. 
3. Patients on blood thinners were excluded.

4. Immuno-compromised patients were excluded.

\subsection{Pre-op protocol}

Patients who conformed to our inclusion criteria, were processed for surgery. Pre-op AP and lateral radiographs were taken of the affected femur. Aneasthetic fitness and written consent were obtained. Second generation cephalosporins were started parentrally 3 to 4 hours prior to surgery. The affected leg and groin till abdomen, were depilated and wrapped in antibiotic solution dipped bandage, prior to being shifted to the OT.

\subsection{Operative Technique}

With the patient supine on the radio-transparent table with sand bags below the ipsilateral pelvis, the affected limb was prepared and draped. Through the lateral approach, non union site was exposed. Rotational instability at the non-union site was visualized. Femoral nail was retained in situ. One $4.5 \mathrm{~mm}$ broad dynamic compression plate was used with $4.5 \mathrm{~mm}$ cortical screws. The intramedullary nail was retained in the static mode and augmentative plating with 6 cortical purchases (bicortical) were secured with 3 proximal and 3 distal to the non-union site (bypassing the intramedullary nail). The proximal end and the distal ends of the fracture sites were freshened and rose petalling of the sub-adjacent diaphyseal bone was done. Liberal quantities of freshly harvested bone graft from the ipsilateral iliac crest were secured circumferentially. Wound was closed in layers over DT and aseptic dressing applied with a long leg compression bandage. The iliac donor site was also closed in layers over a DT, after application of bone wax to the harvested bone site. Parentral antibiotics which were started just before surgical intervention were continued for the next 72 hours.

\subsection{Post-op protocol}

At 72 hours, the DT were removed. Parentral antibiotics were discontinued at 72 hours. However oral antibiotics (Linezolid $600 \mathrm{mg}$ bd and Dalacin C 300mg bd) were continued for 5 more days. Suture removal was done on the POD-12. Patients were taught static quadriceps and straight leg raising exercises from POD-3. Ambulation was allowed from POD-7. Full weight bearing was permitted from the $3^{\text {rd }}$ week onwards. Patients were usually discharged after suture removal and were asked to review once a fortnight for the first two months and on a monthly basis for the next 10 months or until signs of radiological consolidation were seen. After the post-op Xrays, repeat roentgenographic evaluation was done once in 2 months until the 12 months of follow-up or until bony union.

\section{Results}

Of the 24 cases included in this case study there was a male preponderance in the ration of 4:2 (male - 16, female - 8). $65 \%$ patients were in the age group 30 to 39 years and the remaining $35 \%$ of patients were in the 40 to 49 year age group. The time lag between the primary and secondary surgery has been tabulated in table: 1 . The period taken for radiological evidence of sound bony healing has been tabulated in table: 2 . None of the cases went on for non-union. There were $20.8 \%(n=5)$ patients who developed superficial wound infection at the femoral operated site and $8.3 \%(n=2)$ of patients at the iliac crest operated site, all these cases settled with a course of oral antibiotic for 10 days and with regular dressing using silver streak solution (colloidal silver ion solution).
Time 1: lag between primary and secondary surgery.

\begin{tabular}{|c|c|c|c|}
\hline Period in months & Male (n) & Female (n) & Toatal (n) - (\%) \\
\hline 4 & 01 & 01 & $02-(8.4 \%)$ \\
\hline 5 & 10 & 02 & $12-(50 \%)$ \\
\hline 6 & 04 & 01 & $05-(20.8 \%)$ \\
\hline 7 & 00 & 01 & $01-(4.1 \%)$ \\
\hline 8 & 00 & 01 & $01-(4.1 \%)$ \\
\hline 9 & 01 & 02 & $03-(12.6 \%)$ \\
\hline Total & 16 & 08 & $24-(100 \%)$ \\
\hline
\end{tabular}

Table 2: Time taken for evidence of radiological union.

\begin{tabular}{|c|c|c|c|}
\hline Period in weeks & Male (n) & Female (n) & Total (n) - (\%) \\
\hline 20 & 04 & 02 & $06-(25 \%)$ \\
\hline 21 & 01 & 01 & $02-(8.3 \%)$ \\
\hline 22 & 01 & 01 & $02-(8.3 \%)$ \\
\hline 23 & 08 & 04 & $12-(50 \%)$ \\
\hline 24 & 02 & 00 & $02-(8.3 \%)$ \\
\hline
\end{tabular}

\section{Case Illustration 1}

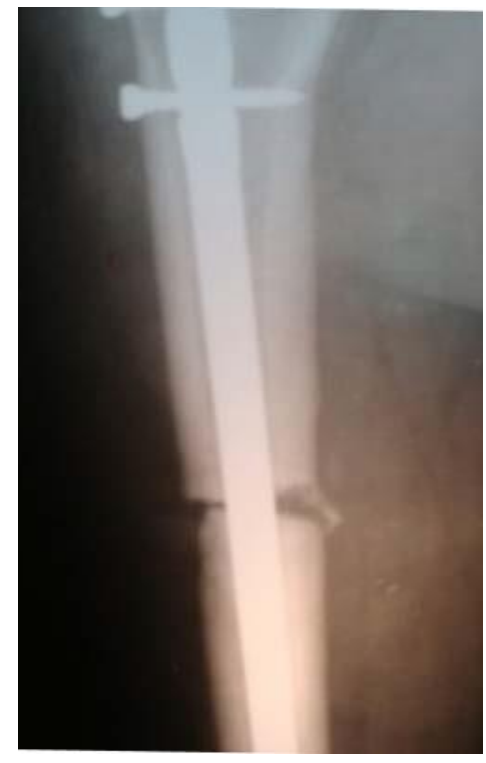

Fig 1(A): Pre-op x-ray showing gap no union with static IMIL femoral nail in situ.

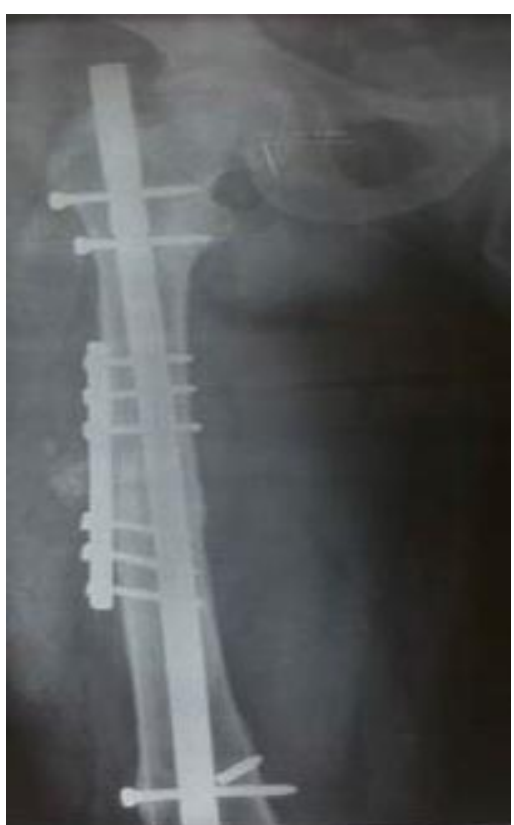

Fig 1(B): Post-op x-ray after plate augmentation at 10 months follow up, showing sound radiological union. 


\section{Case Illustration 2}

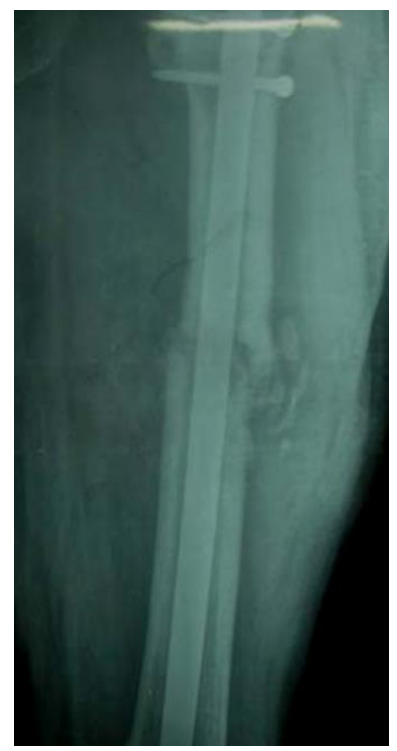

Fig 2(A): Pre op x-ray showing non-union with static IMIL nail in situ.

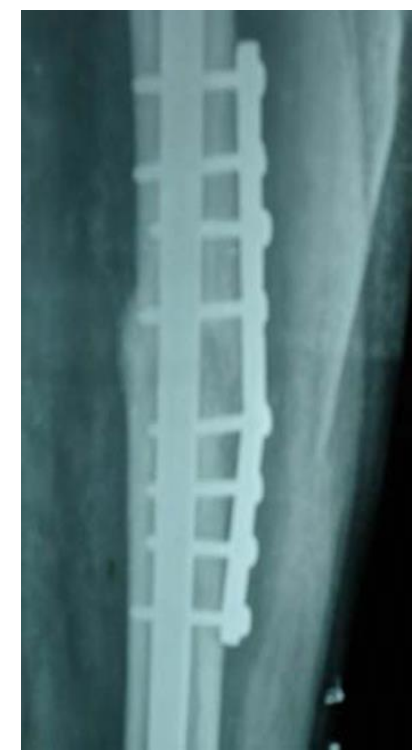

Fig 2(B): Post op x-ray after plate augmentation at 10 month follow up, showing sound radiological union.

\section{Discussion}

Despite recent advancements in fracture treatment, complication like non-union in cases of femoral shaft fractures following intramedullary interlocking nailing are still encountered (15 to $22 \%)^{[3]}$. Reamed exchange nailing has been the treatment of choice in cases of aseptic, noncomminuted non-unions of the femoral diaphysis with intramedullary nail in-situ. In comminuted fractures the intramedullary nails does not fill the canal defect snugly, thus interfering with both bending and rotational stability of the fracture. The wide medullary canal in the distal third of the femoral shaft fractures further compromises the stability of fracture. The effectiveness of plate augmentation and their outcomes had been questioned. Weresh et al. ${ }^{[4]}$ reported that only ten of nineteen $(52.6 \%)$ femoral nonunions following locked intramedullary nailing of comminuted femoral fractures went to osseous union after exchange nailing. Banaszkiewicz et al. ${ }^{[5]}$ reported that only eleven of nineteen (57.8\%) aseptic non-unions following locked intramedullary nailing of high energy, comminuted fractures went on to osseous union after treatment with exchange nailing alone. Augmentative plate fixation in the presence of existing nail has been used for the management of femoral non-unions following intramedullary nailing. All the studies done, which have verified rotational instability of the fracture site during surgery, which was effectively eliminated after plate augmentation. In all our cases we visualised abnormal mobility at the fracture site which was effectively eliminated after plate augmentation.

Comparative studies between nail exchange versus augmentative osteosynthesis have yeilded results favoring the later. This has been confirmed by the studies done by Ueng SW et al ${ }^{[6]}$, Choi YS et al. ${ }^{[7]}$ and Roetman B et al. ${ }^{[8]}$ Our results compares well with their average bone consolidation time of 4 to 6 months.

\section{Conclusion}

Our study shows that augmentative plate osteosynthesis with autologous bone grafting gives excellent mechanical stability and improves the biological environment for fracture healing with less complications and fewer chances for re-surgery. Hence our study concludes that the augmentative plating with autologus bone grafting is a promising alternative to reamed exchange nailing for diaphyseal femoral aseptic non-union. We are also tempted to advocate actually that primary nailing with augmentative plating and bone grafting for the comminutted femoral diaphyseal and diaphyseo-metaphyseal fractures, rather than wait for the non-union to happen and then intervene secondarily.

\section{References}

1. Bhandari M, Guyatt G, Khera V, Kulkamai A, Sprague S, Schemitsch E. Operative management of lower extrimity fractures in patients with head injury. Clin Orthop. 2003; 407:187-98.

2. Nowotarski PJ, Turen CH, Brumback RJ, Scarboro JM. Conversion of external fxation to intramedullary nailing for fractures of shaft of femur in multiple injured patients. J Bone Joint Surg Am. 2000; 82:781-8.

3. Yong-Gang $\mathrm{Ma}$, Ge-Liang $\mathrm{Hu}$, fan Liang. Surgical factors contributing to nonunion in femoral shaft fracture following intramedullary nailing. Chin J Traumatol. 2016; 19(2):109-112.

4. Weresh MJ, Hakanson R, Stover MD. Failure of exchange reamed intramedullary nails for ununited femoral shaft fractures. J Orthop Trauma. 2000; 14(5):335-338.

5. Banaszkiewicz PA, Sabboubeh A, McLeod I. Femoral exchange nailing for aseptic non-union: not the end to all problems. Injury. 2003; 34(5):349-356.

6. Ueng SW, Chao EK, Lee SS. Augmentative plate fixation for the management of femoral non-union after intramedullary nailing. J Trauma. 1997; 43(4):640-644.

7. Choi YS, Kim KS. Plate augmentation leaving the nail insitu and bone grafting for non-union of femoral shaft fractures. Int Orthop. 2005; 29(5):287-290.

8. Roetman B, Scholz N, Muhr G. Augmentive plate fixation in femoral non-unions after intramedullary nailing. Strategy after unsuccessful intramedullary nailing of the femur. Z Orthop Unfall. 2008; 146(5):586590. 